\title{
Fallstricke und Grenzen der Nutzwertanalyse
}

Das Hauptproblem der Nutzwertanalyse ist der Aufwand, der zu tätigen ist. Dieser verführt zuweilen dazu, durch methodische Abkürzungen Zeit sparen zu wollen. Das schlägt regelmäßig fehl! Wird das Verfahren nicht wie beschrieben und mit der gebotenen Sorgfalt durchgeführt, sollte darauf verzichtet werden. Eine „Nutzwertanalyse light“ gibt es nicht. Sie ist auch kein Medikament gegen Entscheidungsfeigheit.

Ein ganz elementarer Erfolgsfaktor der Methode ist die Qualität des Moderators. Er muss das Fachwissen und sollte die nötige Erfahrung besitzen, eine Nutzwertanalyse zu leiten, aber über die Kompetenz und das Auftreten verfügen, die Teilnehmer zu disziplinieren. Seine Aufgabe ist es, über die Methodik zu wachen und sicherzustellen, dass sich alle Teilnehmer einbringen. Bewährt hat sich, klare Spielregeln zu benennen und deren Einhaltung sicher zu stellen. So ist z. B. häufig ein Streitpunkt, welche Kriterien aufgenommen werden. Darüber kann natürlich abgestimmt werden, aber besser ist es, alle sinnvollen aufzunehmen und diese wie beschrieben zu gruppieren.

Doch darf an dieser Stelle nicht versäumt werden, darauf hinzuweisen, dass erfahrungsgemäß die Ergebnisse tendenziell konsensual ausfallen, also einem Mittelwert zustreben. Nur geringe Unterschiede zwischen Handlungsalternativen sind typisch, da die meisten Menschen extreme Bewertungen zu vermeiden suchen. Dennoch ist das Ergebnis mit Sicherheit belastbarer als eines, das sich aus einer von dominanten Figuren geprägten Gruppendiskussion im Rahmen eines „Meetings“ ergäbe. 